\title{
Qualidade de vida em estudantes de Medicina no início e final do curso: avaliação pelo Whoqol-bref
}

PALAVRAS-CHAVE

- Qualidade de Vida

- Estudantes de Medicina

- Estresse
KEY WORDS

- Quality of Life

- Medical Students

- Stress
Recebido em: 11/06/2008 Reencaminhado em: 26/02/2009 Aprovado em: 02/07/2009

REVISTA BRASILEIRA DE EDUCAÇ̃̃O MÉDICA

\section{Quality of life among first and last-year medical students: an evaluation using Whoqol-bref}

João Guilherme Bezerra Alves ${ }^{1}$

Manuela Tenório ${ }^{\mathrm{II}}$

Amanda Gomes dos Anjos ${ }^{\text {II }}$

José Natal Figueroa ${ }^{\mathrm{I}}$

\begin{abstract}
RES U M O
O objetivo do estudo é comparar a qualidade de vida de estudantes de Medicina do primeiro período e do último período do curso. Foi realizado um estudo transversal com a aplicação do instrumento da Organização Mundial da Saúde para avaliação da qualidade de vida no seu formato curto (Whoqol-bref) em 370 estudantes de Medicina da cidade do Recife, Brasil - 229 do primeiro e 141 do último período. Os resultados mostraram que os escores do domínio psicológico foram menores nos alunos do último período do que nos do primeiro período $(\mathrm{p}<0,005)$. Essa diferença se manteve após a análise pelo modelo de covariância (Ancova). Em relação aos escores do domínio físico, relações sociais e meio ambiente, não houve diferenças significativas entre os alunos do primeiro e do último período. Quanto à autoavaliação da qualidade de vida, os alunos do primeiro período apresentaram melhores resultados $(3,87$ vs. 3,39; $\mathrm{p}<0,001)$. Quanto à satisfação com a própria saúde, não houve diferença estatística; 3,94 vs.3,62 ( $p=0,099)$. Em conclusão, a qualidade de vida dos estudantes de Medicina, quando avaliada pelo instrumento Whoqol-bref, sofre desgastes no domínio psicológico durante o curso médico. Novos estudos são necessários para identificar os fatores que determinam essas alterações na qualidade de vida dos estudantes de Medicina durante a graduação.
\end{abstract}

\section{A B S T R A C T}

The aim of this study was to compare the quality of life of first and last-year medical students. A cross-sectional study was performed using the brief format of the World Health Organization instrument for evaluating quality of life (Whoqol-bref) in a sample of 370 medical students from Recife, Pernambuco State Brazil (229 first-year and 141 last-year students). According to the findings, scores from the psychological health domain were lower for last-year as compared to first-year students ( $\mathrm{p}$ 0,005). This difference remained after covariance analysis (Ancova). As for scores in the physical health domain, social relationships, and the environment, there were no significant differences between first and last-year students. For self-rated quality of life, first-year students showed better results (3,87 vs. 3,39; $<<0,001)$. There was no statistically significant difference in health-related self-satisfaction; $3,94$ vs. 3,62 ( $\mathrm{p}=0,099)$. In conclusion, medical students' quality of life declines during medical school as measured by the Whoqol-bref psychological health domain. Further research is needed to identify the factors leading to these changes in quality of life among medical students during their undergraduate training.

\footnotetext{
I Instituto de Medicina Integral Prof. Fernando Figueira, Recife, PE, Brasil.

${ }^{I I}$ Faculdade Pernambucana de Saúde, Recife, PE, Brasil.
} 


\section{INTRODUÇÃO}

A avaliação da saúde, nos últimos anos, vem se preocupando em estabelecer parâmetros mais amplos do que a simples ausência de doenças, com crescente interesse no desenvolvimento de medidas que retratem a "qualidade de vida" das pessoas ${ }^{1,2}$. A qualidade de vida tem sido definida como uma percepção do sujeito em relação a sua posição individual na vida e no contexto de sua cultura e sistema de valores em que está inserido, bem como em relação a seus objetivos, expectativas, padrões e preocupações. É um conceito de alcance abrangente, afetado de forma complexa pela saúde física, estado psicológico, nível de independência, relações sociais e relações com as características do meio ambiente do indivíduo ${ }^{3-6}$. $\mathrm{Na}$ tentativa de uniformizar a avaliação da qualidade de vida, a Organização Mundial da Saúde (OMS) desenvolveu um questionário específico para esse fim, o World Health Organization Questionnaire for Quality of Life-BriefForm (Whoqol-bref) ${ }^{7}$. Esse questionário já foi validado no Brasil ${ }^{8}$.

Vários estudos em diferentes partes do mundo têm mostrado situações estressantes na vida do médico que comprometem sua qualidade de vida ${ }^{9-12}$. Pesquisa recente divulgada pelo Conselho Federal de Medicina revelou que mais da metade dos médicos no Brasil apresenta distúrbios psiquiátricos, como ansiedade e depressão, além de estafa. Também apontou que $5 \%$ dos médicos se sentem sem esperança, infelizes e com pensamentos suicidas ${ }^{13}$. Alguns estudos apontam a presença de fatores estressantes já na formação médica e suas consequências para a saúde dos estudantes ${ }^{14-17}$. Enns et al. ${ }^{18}$ enfatizam que fatores estressantes - como pressão para aprender, grande quantidade de novas informações, falta de tempo para atividades sociais, contato com doenças graves e com a morte no cuidado clínico dos pacientes - podem contribuir para o aparecimento de sintomas depressivos nos estudantes. E Costa e Perei$\mathrm{ra}^{19}$ discorrem sobre os diversos tipos de abuso (verbal, institucional, por risco médico desnecessário, físico e sexual) vivenciados pelos estudantes de Medicina, que podem agravar seu estresse. Como decorrência do estresse, estudos têm demonstrado alta prevalência de suicídio, depressão, uso de drogas, distúrbios conjugais e disfunções profissionais em médicos e estudantes de Medicina que podem prejudicar o cuidado do paciente $\mathrm{e}^{20-24}$.

Não encontramos na literatura consultada, Medline e Scielo, estudos no Brasil que avaliem possíveis mudanças na qualidade de vida dos alunos durante o curso médico. Mesmo no exterior, estudos com essa finalidade são escassos e focados mais na qualidade de vida dos alunos durante o internato ${ }^{25-27}$. Dessa forma, o objetivo deste estudo foi avaliar e comparar a qualidade de vida dos estudantes de Medicina no início e no final do curso, utilizando o instrumento da Organização Mundial da Saúde, o Whoqol-bref.

\section{MÉTODOS}

Trata-se de um estudo descritivo de corte transversal, realizado em Recife, no Estado de Pernambuco, com alunos da Universidade Federal de Pernambuco (UFPE), Universidade Estadual de Pernambuco (UPE) e Escola Pernambucana de Medicina (EPM). Essas três escolas admitem 75, 80 e 120 alunos por semestre, respectivamente.

O estudo foi do tipo censitário, considerando como população a totalidade dos acadêmicos de graduação dos cursos vinculados ao projeto. Entretanto, como foi trabalhada uma amostra do tipo acidental, composta por aqueles que estiveram presentes no momento da coleta de dados, conseguiu-se estudar $83,2 \%$ dos alunos do primeiro semestre e $90,9 \%$ dos alunos do último período do curso de Medicina dessas universidades; o número total de alunos do último semestre é menor porque a EPM iniciou seu curso médico em 2006.

A coleta de dados foi realizada de agosto de 2006 a abril 2007, por meio do questionário Whoqol-bref. Esse questionário é composto por quatro domínios da qualidade de vida, sendo que cada domínio tem por objetivo verificar a capacidade física (sete questões), o bem-estar psicológico (seis questões), as relações sociais (três questões) e o meio ambiente onde o indivíduo está inserido (oito questões), totalizando 24 questões. Além desses quatro domínios, o questionário tem duas perguntas gerais sobre qualidade de vida global. O instrumento Whoqol-bref considera as duas últimas semanas vividas pelos respondentes, e o tempo médio de seu preenchimento é de dez minutos.

As respostas às questões são dadas numa escala com um único intervalo de 0 (zero) a 5 (cinco), segundo a metodologia Whoqol. A equação sugerida pela OMS foi aplicada para a estimativa de escores de cada domínio. Os escores finais de cada domínio são calculados por uma sintaxe, que considera as respostas de cada questão que compõem o domínio, resultando em escores finais numa escala de 4 a 20. Foram excluídos da análise os alunos que não preencheram $80 \%$ do questionário.

O processamento e a análise dos dados foram realizados nos programas estatísticos Stata/SE 9.2 e Microsoft Excel. As questões (1) Como você avalia sua qualidade de vida? (muito ruim; ruim; nem ruim nem boa; boa; muito boa) e (2) Quão satisfeito(a) você está com a sua saúde? (muito insatisfeito; insatisfeito; nem satisfeito nem insatisfeito; satisfeito; muito satisfeito) foram analisadas separadamente, porque não estão incluídas nas equações. Os dados estatísticos foram apresentados em médias e desvios padrões. Foi utilizado o teste "t student" para amostras independentes, com a finalidade de comparar os escores dos alunos em início com os daqueles no final do curso. Para controlar 
um possível efeito de confundimento das variáveis estudadas, foi realizada a análise de covariância (Ancova) para as variáveis que apresentaram significância estatística na análise bivariada.

O estudo teve a aprovação prévia do Comitê de Ética em Pesquisa do Instituto Materno Infantil Prof. Fernando Figueira. Todos os participantes assinaram o termo de consentimento livre e esclarecido.

\section{RESULTADOS}

Foram estudados 370 estudantes, $229(61,8 \%)$ do primeiro ano e 141 (38,1\%) do sexto ano. Dois alunos foram excluídos por não preencherem $80 \%$ do questionário. Não houve diferenças em relação ao sexo, mas a faixa etária foi maior entre os alunos do sexto ano, assim como o número de estudantes casados (Tabela 1).

Quanto à primeira questão - como os acadêmicos se autoavaliavam em relação à qualidade de vida -, os alunos do primeiro período apresentaram melhores resultados: 3,87 vs. 3,39; $\mathrm{p}<$ 0,001 . Em relação à satisfação com a própria saúde, não houve diferença estatística: 3,94 vs. 3,62 ( $\mathrm{p}=0,099)$.

Não houve diferença estatística em relação aos domínios físico, relações sociais e meio ambiente. O domínio psicológico apresentou maiores escores nos alunos do primeiro ano do curso de Medicina em ambos os gêneros (Tabelas 2 e 3). Essa diferença permaneceu estatisticamente significante após o ajuste para as duas variáveis, idade e estado civil, pelo modelo Ancova.

Tabela 1

Descrição e comparação das variáveis demográficas dos estudantes do primeiro e do último período do curso de Medicina, Recife, 2007

\begin{tabular}{lcc}
\hline Variável & $\begin{array}{c}\text { Estudantes do primeiro período } \\
\mathrm{n}=229(\%)\end{array}$ & $\begin{array}{c}\text { Estudantes do último período } \\
\mathrm{n}=141(\%)\end{array}$ \\
\hline Sexo & & \\
$\quad$ Masculino & $96(41,9)$ & $63(44,7)$ \\
$\quad 133(58,1)$ & $78(55,3)$ \\
Feminino & $21,10(2,58)$ & $27,65(1,34)$ \\
Idade média em anos (DP) & & $7(5,0 \%)$ \\
Estado civil & $2(0,9 \%)$ & 0,002 \\
Casado & $227(99,1 \%)$ & $134(95 \%)$ \\
Solteiro & & $0,013^{* *}$ \\
\hline
\end{tabular}

\footnotetext{
* Teste "t student"

** Teste do qui-quadrado
}

\section{DISCUSSÃO}

Em nosso estudo, observamos um decréscimo significativo do domínio psicológico entre os alunos em conclusão do curso médico, quando comparados aos estudantes do início do curso. A hipótese de que seis anos do curso médico possam alterar a qualidade de vida dos estudantes tem sido fundamentada em avaliações de declínio dos domínios psicológicos do estado de saúde. Goldin et al..$^{25}$ observaram diminuição na qualidade de vida e no sono, além de aumento da depressão em estudantes de Medicina durante o internato em cirurgia. Raj et al. ${ }^{26}$ também detectaram uma significativa deterioração nos domínios da vitalidade, saúde física e psíquica ao estudarem prospectivamente a qualidade de vida relacionada à saúde num grupo de estudantes do último ano do curso médico durante dez meses. Recentemente, Hassed et al. ${ }^{27}$, utilizando o questionário Whoqol-bref num estudo de coorte por um ano com estudantes de Medicina, também detectaram piora no domínio psicológico, mas não no domínio físico, dados semelhantes aos nossos resultados.

Guthrie et al. ${ }^{28}$ observaram que o bem-estar psicológico de um grupo de estudantes no final do curso médico dependia do estado de saúde mental do aluno no primeiro ano do curso. Como não realizamos um estudo de coorte, não pudemos testar essa hipótese. Tile et al. ${ }^{29}$ reportaram que os médicos residentes do primeiro ano apresentam significantemente melhores escores de saúde mental, assim como melhor percepção geral de saúde e função social do que os residentes do segundo e terceiro anos. 


\section{Tabela 2}

Comparação da World Health Organization Quality of Life - Brief Form (Whoqol-bref) entre alunos do sexo masculino do primeiro e do último período do curso de Medicina, Recife, 2007

\begin{tabular}{|c|c|c|c|}
\hline Domínios & $\begin{array}{l}\text { Alunos do } 1^{\circ} \text { período } \\
\qquad N=96(\mathrm{DP})^{*}\end{array}$ & $\begin{array}{l}\text { Alunos do } 6^{\circ} \text { período } \\
\qquad N=63(\mathrm{DP})^{*}\end{array}$ & $\mathrm{p}$ \\
\hline Físico & $14,01(2,02)$ & $13,93(1,99)$ & 0,767 \\
\hline Psíquico & $15,22(2,05)$ & $14,33(2,15)$ & 0,001 \\
\hline Relações sociais & $15,30(2,47)$ & $14,79(2,51)$ & 0,080 \\
\hline Meio ambiente & $14,47(2,49)$ & $13,88(2,27)$ & 0,351 \\
\hline
\end{tabular}

* Desvio padrão

Tabela 3

Comparação da World Health Organization Quality of Life - Brief Form (Whoqol-bref) entre alunos do sexo feminino do primeiro e do último período do curso de Medicina, Recife, 2007

\begin{tabular}{|c|c|c|c|}
\hline Domínios & $\begin{array}{l}\text { Alunos do } 1^{\circ} \text { período } \\
\qquad N=133(\mathrm{DP})^{*}\end{array}$ & $\begin{array}{l}\text { Alunos do } 6^{\circ} \text { período } \\
\qquad \mathrm{N}=78(\mathrm{DP})^{*}\end{array}$ & $\mathrm{p}$ \\
\hline Físico & $14,01(2,13)$ & $14,29(1,98)$ & 0,702 \\
\hline Psíquico & $15,32(2,33)$ & $14,20(2,29)$ & 0,001 \\
\hline Relações sociais & $15,66(2,35)$ & $14,99(2,31)$ & 0,098 \\
\hline Meio ambiente & $14,55(2,65)$ & $13,97(2,372)$ & 0,498 \\
\hline
\end{tabular}

* Desvio padrão

É comum a observação de que os estudantes de Medicina, assim como os médicos, apresentam elevadas taxas de afecções mentais ${ }^{5,10,13}$. Ainda que tais estudantes apresentem características psicológicas semelhantes às dos estudantes de outras áreas no início do curso de graduação, o estresse se torna um fator importante com o início do treinamento médico e concorre para a redução do bem-estar psíquico e o aumento da ansiedade, depressão e burnout ${ }^{26,30}$.

Apesar de termos estudado uma grande amostra com elevada taxa de resposta, o que minimiza a possibilidade de viés de seleção, e termos utilizado um instrumento validado internacionalmente (Whoqol-bref), inclusive no Brasil, nosso estudo não deixa de ter limitações metodológicas. Inicialmente, por questões operacionais, seu desenho não pôde ser de um estudo de co- orte prospectivo durante os seis anos do curso médico, o que, pelo fato de estudarmos o mesmo grupo populacional em diferentes momentos, poderia trazer maior confiabilidade aos resultados. Em segundo lugar, embora tenhamos procurado controlar por meio da análise estratificada as variáveis, faixa etária e estado civil, outras possíveis variáveis que poderiam contribuir para a qualidade de vida dos estudantes não foram avaliadas. De qualquer forma, pelo elevado número da casuística e pelo método de coleta de dados ter sido exatamente o mesmo, acreditamos que a principal explicação para os nossos resultados esteja na diferença das etapas - início e conclusão - do curso de graduação em Medicina.

Zonta et al. ${ }^{31}$, em estudo qualitativo, apontaram as seguintes estratégias para reduzir o estresse durante o curso médico: valoriza- 
ção dos relacionamentos interpessoais e de fenômenos do cotidiano; equilíbrio entre estudo e lazer; organização do tempo; cuidados com a saúde, alimentação e sono; prática de atividade física; religiosidade; trabalhar a própria personalidade para lidar com situações adversas; procura por assistência psicológica. Sugerem ainda maior preocupação dos envolvidos na educação médica com a qualidade de vida do estudante de Medicina e o desenvolvimento de estratégias para promovê-la ou que preparem o estudante para lidar com o estresse durante a formação médica.

As precárias condições de trabalho, com jornadas extenuantes, multiplicidade de atividades, desgaste profissional e redução dos salários, vêm sendo apontados pelo Conselho Federal de Medicina (CFM) como as principais causas de adoecimento do médico ${ }^{32,33}$. Nossos dados parecem indicar que, antes da diplomação, o médico sofre prejuízos no domínio psicológico durante a graduação. Isto traz importantes implicações para a saúde futura do médico, uma vez que a mente exerce uma profunda influência sobre a saúde e a qualidade de vida das pessoas. Portanto, esse tema deve ser prioritário para os educadores da área de saúde e as autoridades sanitárias.

Em conclusão, a qualidade de vida dos estudantes de Medicina, quando avaliada pelo instrumento Whoqol-bref, sofre desgastes no domínio psicológico durante o curso médico. Novos estudos, com desenhos de coorte, são necessários para identificar os fatores que determinam essas alterações na qualidade de vida dos estudantes de Medicina durante a graduação.

\section{REFERÊNCIAS}

1. Khanna D, Tsevat J. Health-related quality of life: an introduction. Am J Manag Care. 2007;13(Suppl 9):S218-23.

2. Turkel S, Pao M. Late consequences of chronic pediatric illness. Psychiatr Clin North Am. 2007;30(4):819-35.

3. Kaplan RM, Ries AL. Quality of life: concept and definition. COPD. 2007;4(3):263-71.

4. Lohr KN. Assessing health status and quality-of-life instruments: attributes and review criteria. Qual Life Res. 2002;11(3):193-205.

5. Bullinger M. Assessing health related quality of life in medicine. An overview over concepts, methods and applications in international research. Restor Neurol Neurosci. 2002;20(3-4):93-101.

6. Casas Anguita J, Ramón Repullo Labrador J, Pereira Candel J. Medidas de calidad de vida relacionada con la salud. Conceptos basicos, construccion y adaptacion cultural. Med Clin (Barc). 2001;116(20):789-96.
7. The WHOQOL Group. Development of the World Health Organization WHOQOL-BREF quality of life assessment. Psychol Med. 1998;28(3):551-8.

8. Fleck MPA, Louzada S, Xavier M, Chachamovich E, Vieira G, Santos L, et al. Aplicação da versão em português do instrumento abreviado de avaliação da qualidade de vida "WHOQOL-Bref". Rev Saúde Pública. 2000;34(2):178-83.

9. Linzer M, Visser MR, Oort FJ, Smets EM, McMurray JE, De Haes HC et al. Predicting and preventing physician burnout: results from the United States and the Netherlands. Am J Med. 2001;111(2):170-5.

10. Visser MR, Smets EM, Oort FJ, De Haes HC. Stress, satisfaction and burnout among Dutch medical specialists. CMAJ. 2003;168(3):271-5.

11. McManus IC, Keeling A, Paice E. Stress, burnout and doctors' attitudes to work are determined by personality and learning style: a twelve year longitudinal study of UK medical graduates. BMC Med. 2004;2:29.

12. Schwartzmann L. La calidad de vida de los medicos: estudio de una muestra de medicos del Uruguay.Vertex. 2007;18(72):103-10.

13. Barbosa GA, Andrade EO, Carneiro MB, Gouveia VV. A saúde dos médicos do Brasil. Brasília: CFM; 2007. 220 p.

14. Villanueva T, Haivas I. Studying medicine and quality of life. Student BMJ 2006;14:133 -76.

15. Lee J, Graham AV. Students' perception of medical school stress and their evaluation of a wellness elective. Med Educ. 2001;35:652-9.

16. Millan LR, Souza EM, De Marco OLN, Rossi E. Assistência psicológica ao estudante de medicina. In: Marcondes E, Gonçalves EL, orgs. Educação médica. São Paulo: Savier; 1998. p. 340-54.

17. Supe AN. A study of stress in medical students at Seth G.S.Medical College. J Postgrad Med. 1998;44(1):1-6.

18. Enns MW, Cox BJ, Sareen J, Freeman P. Adaptive and maladaptive perfectionism in medical students: a longitudinal investigation. Med Educ. 2001; 35(11):1034-42.

19. Costa LSM, Pereira CAA. O abuso como causa evitável de estresse entre estudantes de Medicina. Rev Bras Educ Med. 2005;29(3):185-90.

20. Ritson B. Alcohol and medical students. Med Educ. 2001;35(7):622-23.

21. Webb E, Ashton CH, Kelly P, Kamah F. An update on British medical students' lifestyles. Med Educ. 1998;32(3):325-31 
22. Richings JC, Khara GS, Mcdowell M. Suicide in young doctors. Bri J Psychiatry. 1986; 149(4):475-8.

23. Meleiro AMAS. Suicídio entre médicos e estudantes de medicina. Rev Ass Med Bras. 1998;44(2):135-40.

24. Rossetto MAC, Skawinski LSR, Coelho ACP, Rossetto Júnior JA, Bolla K. Avaliação das características psicológicas dos estudantes de medicina por meio do método de rorschach. Psikhe. 2000;5(2):41-51.

25. Goldin SB, Wahi MM, Farooq OS, Borgman HA, Carpenter HL, Wiegand LR, Nixon LL, Paidas C, Rosemurgy AS 2nd, Karl RC. Student quality-of-life declines during third year surgical clerkship. J Surg Res. 2007;143(1):151-7.

26. Raj SR, Simpson CS, Hopman WM, Singer MA. Health-related quality of life among final-year medical students CMAJ. 2000;162(4):515-6.

27. Hassed C, de Lisle S, Sullivan G, Pier C. Enhancing the health of medical students: outcomes of an integrated mindfulness and lifestyle program. Adv Health Sci Educ Theory Pract. 2009;14(3):387-98.

28. Guthrie E, Black D, Bagalkote H, Shaw C, Campbell M, Creed F. Psychological stress and burnout in medical students: a five-year prospective study. J R Soc Med 1998; 91(5):237-43.

29. Tile L, Singer M, Simpson C, Hopman W. Health status assessment of postgraduate trainees in Internal Medicine. Ann R Coll Physicians Surg Can. 1995;28:403-6.

30. Dyrbye LN, Thomas MR, Shanafelt TD. Systematic review of depression, anxiety, and other indicators of psychologi- cal distress among U.S. and Canadian medical students. Acad Med. 2006; 81(4):354-73.

31. Zonta R, Robles ACC, Grosseman S. Estratégias de enfrentamento do estresse desenvolvidas por estudantes de medicina. Rev Bras Educ Med. 2006;30(3):147-53.

32. Machado MH, coord. Perfil dos médicos no Brasil. Rio de Janeiro: FIOCRUZ;CFM-MS;PNUD; 1996.

33. Barbosa GA, Andrade ED, Carneiro MB, Gouveia VV. A saúde dos médicos no Brasil. Brasília: CFM; 2007. 220p.

\section{CONTRIBUIÇÃO DOS AUTORES}

João Guilherme Bezerra Alves desenhou o projeto e redigiu o trabalho; Manuela Tenório e Amanda Gomes dos Anjos participaram da coleta e análise dos dados; José Natal Figueroa participou da análise dos resultados e redação final.

\section{CONFLITO DE INTERESSES}

Declarou não haver.

\section{ENDEREÇO PARA CORRESPONDÊNCIA}

Instituto de Medicina Integral Prof. Fernando Figueira (IMIP)

Rua dos Coelhos, 300

Boa Vista - Recife

CEP. 50070-550 PE

E-mail: joaoguilherme@imip.org.br 